\title{
Breaking up-down symmetry with magnetic perturbations in tokamak plasmas: increase of axisymmetric steady-state velocities
}

\author{
H. Oueslati ${ }^{1,2}$ and M.-C. Firpo ${ }^{1, a)}$ \\ ${ }^{1)}$ Laboratoire de Physique des Plasmas, CNRS - Ecole Polytechnique, PSL Research University, \\ 91128 Palaiseau cedex, France \\ ${ }^{2)}$ Département de physique, Faculté des Sciences, Université de Tunis el Manar, \\ Tunisia
}

( Dated: 17 August 2020)

Plasma rotation plays a crucial role on heat and particle confinement in tokamaks. To consider this issue, we numerically compute the axisymmetric steady states of the visco-resistive magnetohydrodynamic equations including the non-linear $(\mathbf{v} \cdot \nabla) \mathbf{v}$ term using the finite element method. Imposing external $n=0$ magnetic perturbations offers a way to break the natural up-down symmetry of the system and produce a net toroidal flow. Using realistic parameters, some numerical results indicate that $n=0$ perturbations of the magnetic configuration may be used to increase $n=0$ steady-state speeds and promote tokamak plasma confinement whilst preserving axisymmetry.

\section{INTRODUCTION}

It has long been recognized in the magnetic confinement fusion community that plasma rotation plays an important role on the heat and particle confinement properties $^{1}$. This raises naturally the question of the derivation of steady-state flows in tokamak plasmas. The usual assumption made by linear magnetohydrodynamic (MHD) theory is to neglect them which allows also to make less intricate its analytical derivation ${ }^{2}$. However, it was shown ${ }^{3}$ that, although there are rigorously motionless steady-states in cylindrical geometry, this can no longer be true in toroidal geometry. Because of toroidal geometry, resistive steady-state flows of tokamak plasmas are non-vanishing. There is then, at least, some fundamental interest in the investigation of steady-states in the fluid, MHD, description of plasmas in toroidal devices for magnetic confinement fusion. There is also some pragmatic interest in terms of potential applications in the search of conditions enhancing steady-state plasma speeds, when viewing the fluid MHD description as a first step towards the more realistic and complex (gyro)kinetic description of future burning plasmas in magnetic confinement fusion.

Then one needs to evaluate the minimal necessary ingredients to put in the MHD description of tokamak plasmas. These are devices driven by two external, curlfree, toroidal magnetic and electric fields. The later one produces the toroidal current that is the source of the poloidal magnetic field. This driving introduces naturally two parameters in the model. As for the equations themselves, one retains a visco-resistive description in toroidal geometry. This introduces two other (small) dimensionless parameters: the resistivity $\eta$ and the kinematic viscosity $\nu$. These are respectively the inverses of the magnetic Reynolds, $S$, and viscous Lundquist, $M$,

a)Electronic mail: firpo@lpp.polytechnique.fr numbers. The Hartmann number, $H \equiv \sqrt{M S}$, happens to be a meaningful combination of those numbers. This was the setting chosen by Kamp and Montgomery ${ }^{4}$ who first investigated the visco-resistive MHD steady-states for tokamaks using idealistic up-down symmetric plasma domains. Later the effect of having a tokamak with a cross-section that is not symmetric about the midplane ('D-shaped') was considered ${ }^{5,6}$. A net toroidal mass flow can then develop. However, the maximum velocities, in the region where the viscosity was large enough for the numerics to work, were of the order of a few meters per second, far below the speeds commonly measured in tokamak plasmas. Recently, a FreeFem $++{ }^{7}$ code was developed to solve the axisymmetric steady-state flows in the visco-resistive frame relevant to tokamak physics using JET and ITER geometries. The obtained results ${ }^{8}$ confirm that, within the visco-resistive MHD frame and for the numerically manageable viscosity values, these $n=0$ speeds are far below the levels expected to allow the stabilization of MHD modes and positively impact confinement.

In the present study, the symmetry properties with respect to the tokamak midplane of the visco-resistive MHD system are analyzed. Considering tokamaks with up-down symmetric cross-sections, the possibility of playing on boundary conditions to break symmetry and allow a net toroidal mass flow is investigated through the use of external magnetic perturbations. In Section II, the essentials of the visco-resistive modeling frame relevant to tokamak plasma physics are presented and the up-down symmetry features of the system of differential equations are analyzed. We use the open-source partial differential equations solver FreeFem $++^{7}$ for the numerical resolution of their weak formulation. In Section III, the various physical parameters are discussed. Numerical results are presented showing the impact of external magnetic perturbations on the plasma poloidal and toroidal speeds. In Section IV, JET-relevant physical parameters are used. Robust numerical results up to high Hartmann numbers are obtained for small external $n=0$ magnetic perturba- 
tions. These numerical results indicate that rather high plasma speeds may be reached at fusion-relevant high Hartmann numbers. Conclusions and perspectives are drawn in Section V.

\section{MODELING FRAME}

\section{A. The dimensionless model}

The computation of visco-resistive steady states involves the resolution of the steady-state incompressible Navier Stokes equation (1a)-(1b), including the $(\mathbf{v} \cdot \nabla) \mathbf{v}$ term, coupled to the Ohm's law (1f) and steady-state Maxwell equations for the electromagnetic field (1c)-(1e)

$$
\begin{aligned}
(\mathbf{v .} \nabla) \mathbf{v} & =\mathbf{J} \times \mathbf{B}-\nabla p+\nu \nabla^{2} \mathbf{v} \\
\nabla . \mathbf{v} & =0 \\
\nabla . \mathbf{B} & =0 \\
\nabla \times \mathbf{E} & =0 \\
\nabla \times \mathbf{B} & =\mathbf{J} \\
\mathbf{E}+\mathbf{v} \times \mathbf{B} & =\eta \mathbf{J} .
\end{aligned}
$$

We restrict here to the axisymmetric 2D problem. Variables are made dimensionless in the usual Alfvèn units so that velocities are normalized by the characteristic Alfvèn velocity $v_{\mathrm{A}}=\left(B^{2} / \mu_{0} \rho\right)^{1 / 2}$ where $\rho$ is plasma mass density.

For a detailed presentation of the framework and derivation of the final set of equations (4), the Reader is referred to Refs. ${ }^{4,8}$. Importantly, both the pressure and the electric potential being passive scalars, the knowledge of which are not necessary in solving the model, do not appear there. Moreover, the problem involves four divergence-free fields: the velocity field $\mathbf{v}(r, z)$, the magnetic field $\mathbf{B}(r, z)$, the vorticity field $\boldsymbol{\omega}(r, z)$ and the current density field $\mathbf{J}(r, z)$. In order to enforce the divergence-free properties, scalar potentials are used together with the request for axisymmetry. One defines then by $\psi$ the velocity stream function and by $\chi$ the magnetic flux function. This means that the velocity and magnetic fields read

$$
\begin{aligned}
\mathbf{v}(r, z) & =\nabla \psi \times \nabla \varphi+v_{\varphi} \widehat{\mathbf{i}}_{\varphi} \\
\mathbf{B}(r, z) & =\nabla \chi \times \nabla \varphi+\left(B_{0} \frac{r_{0}}{r}+B_{\varphi}\right) \widehat{\mathbf{i}}_{\varphi} .
\end{aligned}
$$

Similarly, the scalars $r B_{\varphi}$ (diamagnetic function) and $r v_{\varphi}$ play the roles of flux functions for respectively the current density and vorticity fields.

Defining by $r_{0}$, the tokamak major radius, the model
(1) can be finally expressed in terms of six variables

$$
\begin{aligned}
& u_{1}=\frac{\psi}{r_{0}} \\
& u_{2}=r_{0} r \omega_{\varphi} \\
& u_{3}=\frac{r B_{\varphi}}{I_{b}}+1 \\
& u_{4}=\frac{r v_{\varphi}}{I_{b}} \\
& u_{5}=\frac{\chi}{r_{0}} \\
& u_{6}=r_{0} r J_{\varphi}-I_{e}
\end{aligned}
$$

where $I_{b}=r_{0} B_{0}$ and $I_{e}=r_{0}^{2} E_{0} / \eta$. Here $B_{0}$ and $E_{0}$ (alternatively $I_{b}$ and $I_{e}$ ) are the two driving parameters mentioned in the introduction, respectively the values of the external magnetic and electric fields at $r_{0}$.

Space variables are rescaled by $r_{0}$ and one defines the horizontal and vertical coordinates by $x=r / r_{0}$ and $y=$ $z / r_{0}$. The system of equations to be solved reads finally

$$
\begin{aligned}
\triangle^{*} u_{1} & =-u_{2} \\
\nu \triangle^{*} u_{2} & =\frac{I_{b}^{2}}{x^{2}} \frac{\partial u_{3}^{2}}{\partial y}-2 \frac{u_{6}+I_{e}}{x^{2}} \frac{\partial u_{5}}{\partial y} \\
& +\frac{1}{x}\left(\left\{u_{6}, u_{5}\right\}+\left\{u_{1}, u_{2}\right\}\right)+2 \frac{u_{2}}{x^{2}} \frac{\partial u_{1}}{\partial y}-I_{b}^{2} \frac{\partial}{\partial y}\left(\frac{u_{4}^{2}}{x^{2}}\right) \\
\eta \triangle^{*} u_{3} & =\frac{2}{x^{2}}\left(u_{3} \frac{\partial u_{1}}{\partial y}-u_{4} \frac{\partial u_{5}}{\partial y}\right)+\frac{1}{x}\left(\left\{u_{1}, u_{3}\right\}+\left\{u_{4}, u_{5}\right\}\right) \\
\nu \triangle^{*} u_{4} & =\frac{1}{x}\left(\left\{u_{3}, u_{5}\right\}+\left\{u_{1}, u_{4}\right\}\right) \\
\triangle^{*} u_{5} & =-u_{6}-I_{e} \\
\eta u_{6} & =\left\{u_{5}, u_{1}\right\} .
\end{aligned}
$$

In the equations (4), the operator $\triangle^{*}$ is defined by

$$
\triangle^{*} \equiv \frac{\partial^{2}}{\partial x^{2}}-\frac{1}{x} \frac{\partial}{\partial x}+\frac{\partial^{2}}{\partial y^{2}},
$$

and the Poisson bracket $\{\cdot, \cdot\}$ for any space functions $u$ and $v$ by

$$
\{u, v\}=\frac{\partial u}{\partial x} \frac{\partial v}{\partial y}-\frac{\partial u}{\partial y} \frac{\partial v}{\partial x}
$$

\section{B. Domain and boundary conditions}

In order to solve the above steady-state visco-resistive MHD equations, two ingredients must be documented: we must know the geometry of the tokamak plasma domain and the boundary conditions for the unknowns to be fulfilled on its border.

As it is well-known, solving the equilibrium of a plasma is generally a free-boundary problem since the plasma domain is not a priori known, apart from a limiter configuration. The interface between the plasma and the vacuum needs to be obtained by another non-linear problem 
in the vacuum for given currents in external coils. We make the usual simplifying assumption ${ }^{9}$ that the later problem has been solved separately so that we can prescribe the shape of the plasma boundary $\partial \Omega$. In the numerics, we shall use the JET geometry. The parametric equations modeling the boundary $\partial \Omega$ of the plasma cross-section $\Omega \subset \mathbb{R}^{2}$ that generates the full plasma domain under a toroidal rotation are given by

$$
\begin{aligned}
& x=1+\frac{r_{1}}{r_{0}} \cos (\theta+\arcsin \delta \sin \theta), \\
& y=\frac{r_{2}}{r_{0}} \sin \theta,
\end{aligned}
$$

for $\theta \in[0 ; 2 \pi]$. Here $r_{0}$ is the major radius, $r_{1}$ the semi-minor axis and $r_{2}$ the semi-major axis of the crosssection, and $\delta$ the triangularity parameter. Typical JET parameters have been used: the major radius is $r_{0}=3 \mathrm{~m}$, the semi-minor axis radius $r_{1}=1.25 \mathrm{~m}$, the plasma elongation $\kappa=r_{2} / r_{1}=1.55$ and triangularity is such that $\arcsin \delta=0.5$.

We are then left with the prescription of boundary conditions. The system (4) amounts to five coupled secondorder elliptic differential equations for $u_{1}$ to $u_{5}$ so that we need boundary conditions for these five variables. Because $\mathbf{B}, \mathbf{J}, \mathbf{v}$ and $\boldsymbol{\omega}$ are divergence-free, we need to ensure the continuity of the normal component of those vector fields at the boundary $\partial \Omega$. This will give the boundary conditions for the four flux functions-like, respectively $\chi\left(u_{5}\right), r B_{\varphi}\left(u_{3}\right), \psi\left(u_{1}\right)$ and $r v_{\varphi}\left(u_{4}\right)$. Under the assumption that there is no plasma flow outside $\Omega$, the normal components of $\mathbf{v}$ and $\boldsymbol{\omega}$ need to vanish on $\partial \Omega$, which is ensured by taking ${ }^{10} \psi=0$ and $r v_{\varphi}=0$ on $\partial \Omega$. We shall prescribe similarly that the normal component of the current density at the plasma boundary be zero, which is ensured by taking $r B_{\varphi}=0$ on $\partial \Omega$.

Putting aside for a moment the fate of the continuity of the normal component of the magnetic field, we are left with the boundary condition for the toroidal component of the vorticity (variable $u_{2}$ ). We take here the convenient choice that this vanishes on $\partial \Omega$.

The remaining boundary condition involves the continuity of the normal component of the magnetic field. When the plasma is assumed to be in contact with the tokamak wall assumed to be a perfect conductor, then this condition is prescribed by requiring that the potential $\chi$ be constant on the wall. In the present study, we consider that some vacuum surrounds the plasma such that we just request the continuity and continuous derivability $\left(\mathcal{C}^{1}\right.$ character $)$ of the magnetic flux function $\chi$ on $\partial \Omega$. This introduces extra freedom in the problem, since we can study the impact of stationary magnetic perturbations coming from external coils. This is the subject of the present article.

Let us then summarize the boundary conditions in terms of the set of variables $\left\{u_{1}, \ldots, u_{5}\right\}$. We have

$$
\begin{aligned}
& u_{1}=u_{2}=0, u_{3}=1 \text { on } \partial \Omega, \\
& u_{4}=0, u_{5}=\beta(x, y) \text { on } \partial \Omega,
\end{aligned}
$$

where $\beta(x, y)$ denotes some $\mathcal{C}^{1}$ function associated to some external (vacuum) stationary magnetic perturbation. Let us note that this amounts to a no-slip condition only for the toroidal flow. Imposing a no-slip condition also on the poloidal flow would require replacing the condition on $\omega_{\varphi}$ by a condition enforcing the vanishing of the normal derivative of $\psi$ at the border. Yet, this "full" noslip boundary condition is presumably not realistic in the case when the plasma is surrounded by some vacuum and not by material walls. Moreover, we shall see in the sequel that allowing some halo plasma outside $\Omega$ enables to relax the no-slip condition on the toroidal flow may promote much larger steady-state plasma speeds at high Hartmann numbers.

\section{Up-down symmetry features}

Let us examine now the symmetry properties with respect to the horizontal mid-plane $y=0$ of the set $u_{1}, \ldots$, $u_{6}$ solving the system of equations (4). The geometry of the problem is depicted on Figure 1.

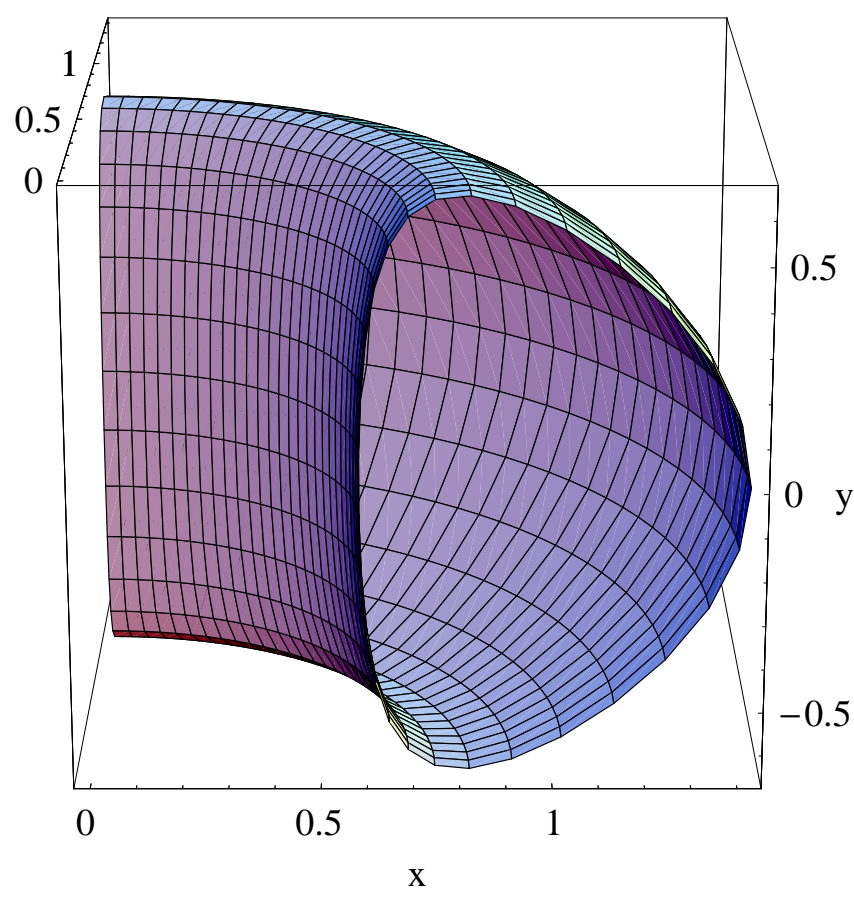

FIG. 1. One-fourth of the full plasma domain. Because axisymmetry is assumed, the problem is invariant by rotation along the toroidal angle so that we can restrict to the $2 \mathrm{D}$ $(x, y)$ cross-section $\Omega$.

As $\triangle^{*}$ is invariant under the change $y \mapsto-y$, from Eqs. (4a) and (4f), we obtain that $u_{1}$ and $u_{2}$ have the same parity (with respect to $y$ ) and so do $u_{5}$ and $u_{6}+I_{e}$. This induces that the right part of the equality (4c) is odd, as the sum of odd terms (such as $\left\{u_{6}, u_{5}\right\}$ ), so that $u_{2}$ (and also $u_{1}$ ) is also odd due to Eq. (4c). Because of 
Eq. (4g), $u_{1}$ and $u_{2}$ do not have the same parity as $u_{5}$ and $u_{6}: u_{5}$ and $u_{6}\left(+I_{e}\right)$ are even functions of $y$. From Eqs. (4d) and (4e), it follows that $u_{3}$ and $u_{4}$ have opposite parities. As $u_{3}$ is defined up to a constant, it cannot be odd so that $u_{4}$ is odd and $u_{3}$ is even.

These symmetry properties with respect to $y$ are preserved when the plasma domain $\Omega$ is symmetric with respect to the $y$-axis (which occurs for the configuration considered here) and for compatible boundary conditions. This is satisfied for the case $\beta(x, y)=0$ in the boundary conditions (8). In this case, the toroidal velocity field $v_{\varphi}$ is an odd function in $y$ so that there is a zero net mass flow in the toroidal direction. In Section III, we address this case first then study the impact of breaking the up-down symmetry by stationary vacuum magnetic perturbations. The numerical resolution of the weak form of the set of partial differential equations (pde) (4) uses the implementation of the NewtonRaphson method ${ }^{8}$ in the pde solver FreeFem ++ with the finite element method.

\section{NUMERICAL RESULTS}

\section{A. Physical parameters}

In the present study, the impact of axisymmetric external magnetic perturbations on the plasma velocity field is investigated by comparison to the reference unperturbed case all other things being equal. This means that we consider the impact of replacing the boundary condition on the magnetic flux function variable $u_{5}=0$ by $u_{5}=\beta(x, y)$ with a small enough norm $\|\beta\|_{2}$ for the same plasma domain $\Omega$, for the same other boundary conditions and parameters. It happens that fully realistic parameters are numerically challenging, which is a wellknown issue in MHD computations for magnetic confinement fusion applications. This will be discussed later in Section IV.

Geometrical JET parameters have been taken in the equations for the plasma border (7) defining the computational domain $\Omega$. The value of the external toroidal magnetic field $B_{0}$ is taken to be $2.8 \mathrm{~T}$. The toroidal loop voltage is chosen to be equal to 1 Volt. This is a reasonable assumption since the toroidal electric field $E_{0}$ is of the order of some V.m ${ }^{-1}$ in the present devices ${ }^{11}$. In dimensionless units, the corresponding parameters are $B_{0}=0.87$ and $E_{0}=3.10^{-9}$. Velocities are expressed in Alfvénic units with a JET-relevant Alfvén velocity $v_{A}=3.10^{6} \mathrm{~m} \cdot \mathrm{s}^{-1}$.

Plasma resistivity, $\eta$, decreases strongly with temperature as $T^{-3 / 2}$ (Spitzer's law ${ }^{12}$ ). As discussed by Kamp and Montgomery ${ }^{5}$, there is some uncertainty or latitude on this parameter. Indeed, if one requires the toroidal current to be equal to $3.1 \mathrm{MA}$ in JET given the previous toroidal loop voltage, this gives by Ohm's law a value of dimensionless resistivity of about $10^{-8}$. Yet if one considers that the JET plasma is made of deuterium, with an electron and ion density both of $3.6 \times 10^{13} \mathrm{~cm}^{-3}$, with an electron temperature of $10 \mathrm{keV}$ and an ion temperature of $18 \mathrm{keV}$, tabulated formulas for transport coefficients indicate that $\eta$ should be two orders of magnitude smaller, of the order $10^{-10}$. This discrepancy is presumably due to anomalous transport. In the simulations we have considered a fixed resistivity with either $\eta=6.9 \times 10^{-7}$ or $\eta=5.5 \times 10^{-9}$. For the later value, that is likely to be more realistic for fusion conditions, the numerical code experiences some stability issues above some critical value of the Hartmann number that is smaller than for the former one. Yet, interestingly, in the presence of external stationary magnetic perturbations, the code appears to become more stable at high Hartmann numbers.

As we have seen, there is some uncertainty on the value of the tokamak plasma resistivity, due to anomalous transport, which can be somehow resolved by asking that the effective resistivity gives the correct value of the toroidal plasma current given the toroidal loop voltage in the application of Ohm's law (when neglecting the $\mathbf{v} \times \mathbf{B}$ term). Uncertainties on the magnitude of the magnetized plasma kinematic viscosity are much higher $^{4,5}$ so that we shall consider it as a free parameter. Given the relevance of the Hartmann number in magnetized plasma channel flows, we shall practically run simulations for a fixed resistivity $\eta$ but for diverse values of the plasma kinematic viscosity $\nu$, so that a wide range of orders of the Hartmann number $H=(\eta \nu)^{-1 / 2}$ be swept. We expect the physically relevant values of $H$ to be large. Their estimation relies on some assumptions on the scalar approximation of the tensor expression for Braginskii-Balescu viscosity coefficients, since a direct tensor description would be too difficult to handle directly. It was discussed ${ }^{4}$ that depending on whether the kinematic viscosity is dominated by either the ion parallel or transverse contribution, the corresponding Hartmann number varies by a factor $10^{6}$ reaching values of the order $10^{8}$ for $\eta=5 \times 10^{-9}$ in the later case.

We shall now start by considering the results obtained in the absence of external magnetic perturbations.

\section{B. Numerical results in the absence of external magnetic perturbations}

As a result of toroidal geometry, some non-vanishing velocity field is needed in the tokamak plasma steadystate equations of motion. The zero-velocity states are compatible with cylindrical geometry but cannot survive the introduction of toroidal effects ${ }^{3}$ so that the traditional Grad-Shafranov equation for tokamak equilibria is -strictly speaking- only approximate. Following a preliminary communication ${ }^{8}$, we examine here results obtained when putting $\beta(x, y)=0$ in the boundary conditions (8b). This corresponds, in particular, to the limiter configuration when the wall is a perfect conductor.

We take the following dimensionless parameters $E_{0}=$ $3 \times 10^{-9}, \eta=6.9 \times 10^{-7}$, take $B_{0}=2.8 \mathrm{~T}$ and compute 


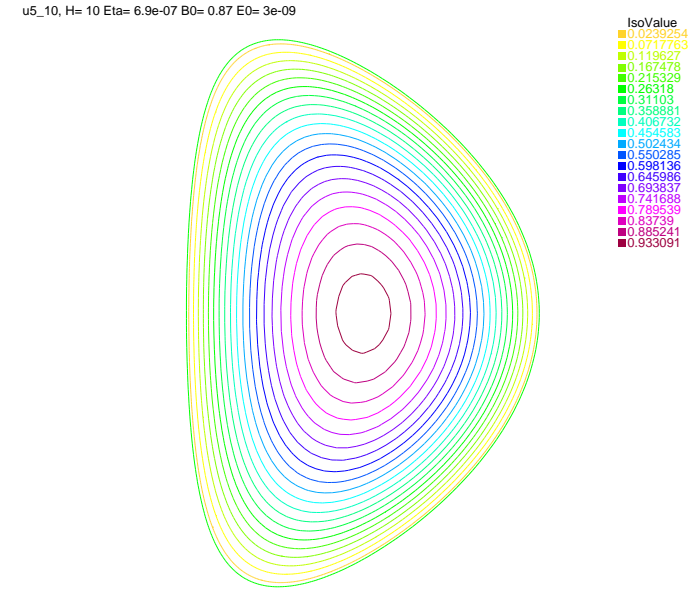

FIG. 2. Contour plot of the magnetic flux function $\chi$ at $H=$ 10 for $E_{0}=3 \times 10^{-9}, \eta=6.9 \times 10^{-7}$, and $B_{0}=2.8 \mathrm{~T}$, with $\chi$ normalized to one on the magnetic axis.

the visco-resistive MHD steady-states using a continuation method on $H$. Magnetic surfaces are represented on Figure 2. These are obtained numerically in the code and are practically unchanged in the range of Hartmann numbers that is covered in this study.

The computed steady-state toroidal velocity fields are presented for Hartmann numbers $H=10, H=10^{4}$ and a large value $H=10^{7}$ on Figs. 3,4 and 5. In terms of

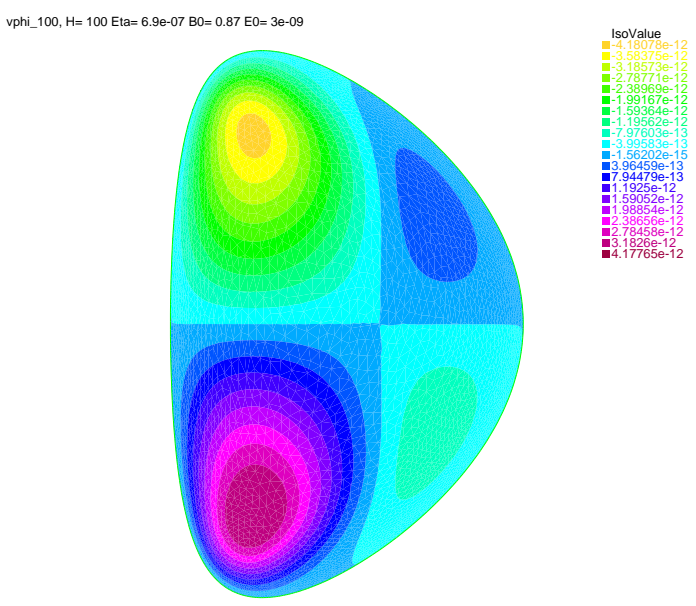

FIG. 3. Toroidal velocity field computed with the Finite Element Method (FEM) using FreeFem ++ with P1 elements for $H=100$ for $E_{0}=3 \times 10^{-9}, \eta=6.9 \times 10^{-7}$, and $B_{0}=2.8 \mathrm{~T}$ in JET geometry with boundary conditions given by (8) with $\beta(x, y)=0$ in $(8)$.

orders of magnitude, these numerical results with symmetric boundary conditions without external magnetic perturbations are in the same range as those obtained by Kamp and Montgomery ${ }^{4}$ (who considered the $\beta(x, y)=0$ case). The natural steady flows obtained in the present simulations happen to be far too small to impact plasma confinement and to account for the measured plasma in-

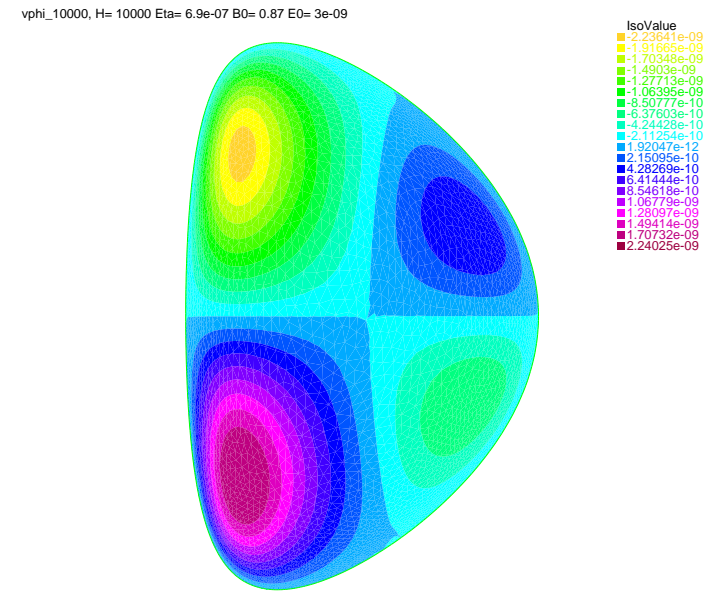

FIG. 4. Same as Fig. 3 for $H=10^{4}$.

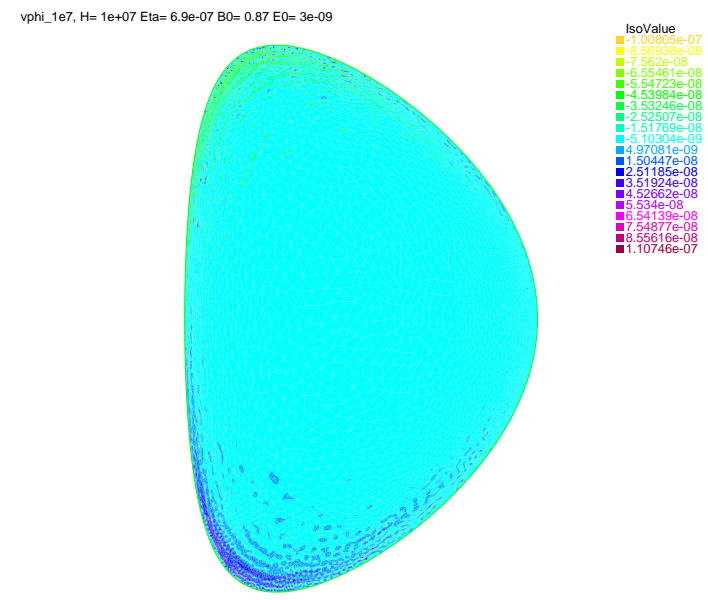

FIG. 5. Same as Fig. 3 for $H=10^{7}$.

trinsic toroidal velocities of the order of some $\mathrm{km} . \mathrm{s}^{-1}$. Indeed, Figure 6 depicts the dependence with $H$ of some averages on the plasma section domain $\Omega$ of the velocity field. The up-down anti-symmetry of the solution for the toroidal flow $\left(v_{\varphi}(x, y)=-v_{\varphi}(x,-y)\right)$ reflects in the negligible value of the absolute value of the mean toroidal velocity $\left\langle v_{\varphi}\right\rangle$ with respect to its root-mean square up to large values of the Hartmann numbers. We expect that symmetry-breaking boundary conditions may offer a way to alleviate this problem by inducing larger steady flows. For this matter, we introduce in the following stationary magnetic perturbations.

\section{Numerical results with external magnetic perturbations}

We now slightly perturb the magnetic configuration by taking into account the effect of small axisymmetric external stationary magnetic perturbations. Practically speaking, we allow $\beta(x, y)$ to be a small function of $x$ and $y$. An example of the magnetic surfaces in the perturbed 


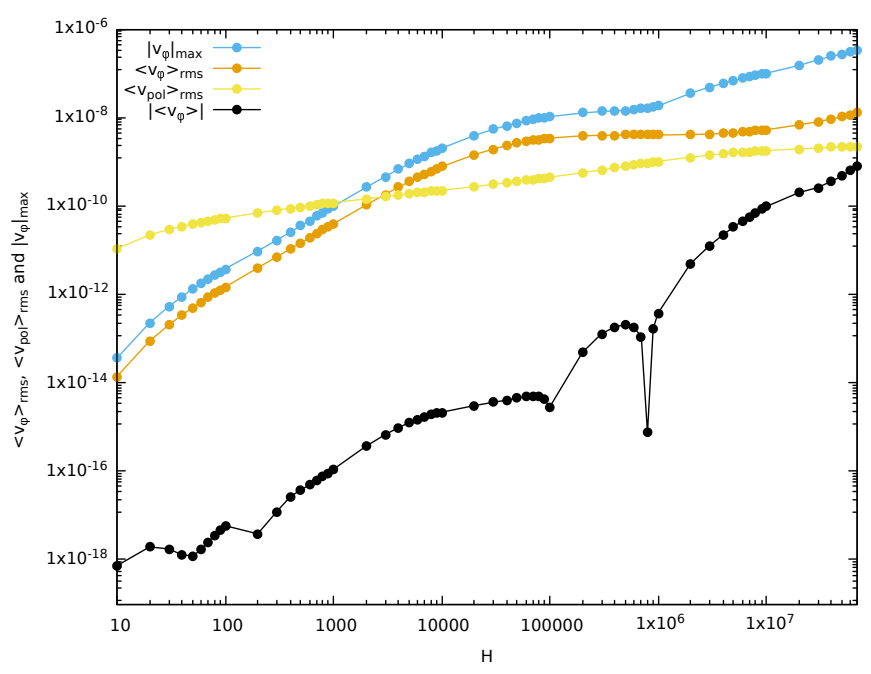

FIG. 6. Maximal absolute value of the toroidal component of the velocity, $\left|v_{\varphi}\right|_{\max }$, and some section-averaged quantities: root-mean square of the toroidal and poloidal velocity, $\left\langle v_{\varphi}\right\rangle_{\mathrm{rms}}$ and $\left\langle v_{\mathrm{pol}}\right\rangle_{\mathrm{rms}}$, absolute value of the average toroidal velocity $\left\langle v_{\varphi}\right\rangle$ associated to the steady flow as a function of the Hartmann number in Alfvèn velocity $v_{\mathrm{A}}$ units.

case is given in Figure 7.

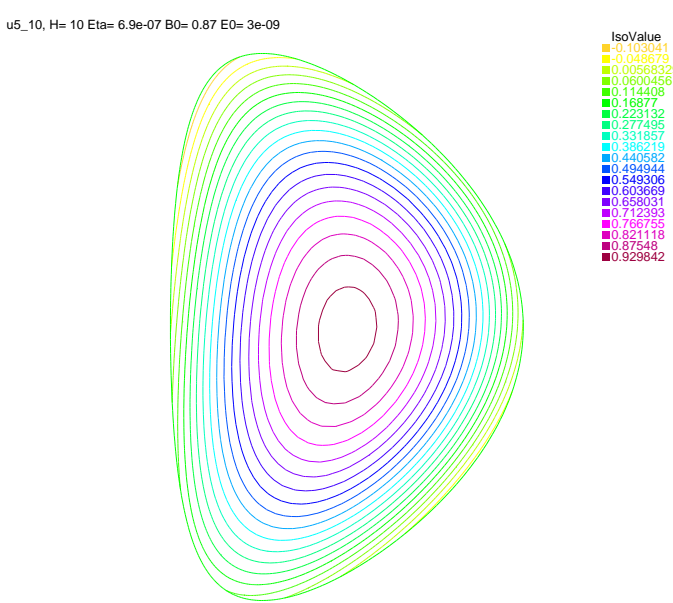

FIG. 7. Contour plot of the magnetic flux function $\chi$ at $H=$ 10 for $E_{0}=3 \times 10^{-9}, \eta=6.9 \times 10^{-7}$, and $B_{0}=2.8 \mathrm{~T}$, in the case $\beta(x, y)=0.001 \sin (x-1) \sin (2 y) . \chi$ is normalized to one on the magnetic axis.

We ran our FEM code for various forms, $f$, and amplitudes, $h$, of $\beta(x, y)$ where we use the writing $\beta(x, y)=$ $h f(x, y)$ with $\|f\|_{2}=\mathcal{O}(1)$ and $h \ll 1$. For the present discussion, we concentrate mostly on three representative cases: $f_{1}(x, y)=\sin (x-1), f_{2}(x, y)=\sin (2 y)$ and $f_{3}(x, y)=\sin (x-1) \sin (2 y)$. Let us note that we could alternatively use the decomposition of these functions $f$ on poloidal $m$ modes. Yet, we find it clearer to maintain $x$ and $y$ variables which are the most suitable to discuss up-down tokamak symmetry features.

Figures 8 and 9 present numerical results on the ve- locity field respectively for the $f_{1}$ and $f_{3}$ cases, with $\beta(x, y)=0.001 \sin (x-1)$ and $\beta(x, y)=0.001 \sin (x-$ 1) $\sin (2 y)$. Insets of the toroidal velocity field on the plasma cross-section $\Omega$ are inserted to show its topology at different Hartmann numbers. The boundary condition with the form $f_{2}$ will be treated in detail afterwards.

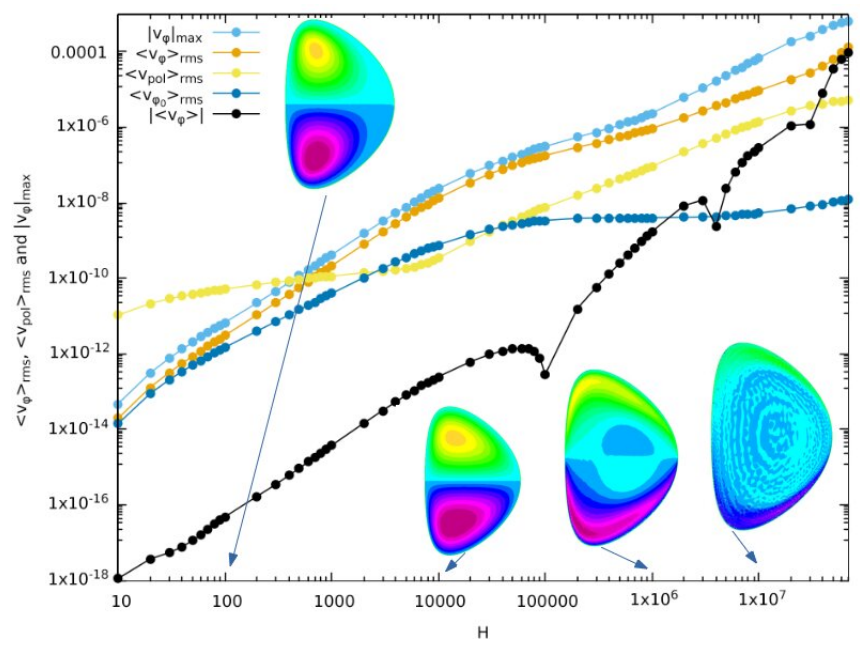

FIG. 8. Same as Figure 6 for the case $\beta(x, y)=0.001 \sin (x-$ $1)$. Some insets of the associated toroidal velocity field on the plasma cross-section $\Omega$ are given for the Hartmann numbers: $H=100, H=10^{4}, H=10^{6}$ and $H=10^{7}$. For comparison, the root-mean square of the toroidal velocity of the $\beta=0$ case, $\left\langle v_{\varphi 0}\right\rangle_{\mathrm{rms}}$ has been plotted.

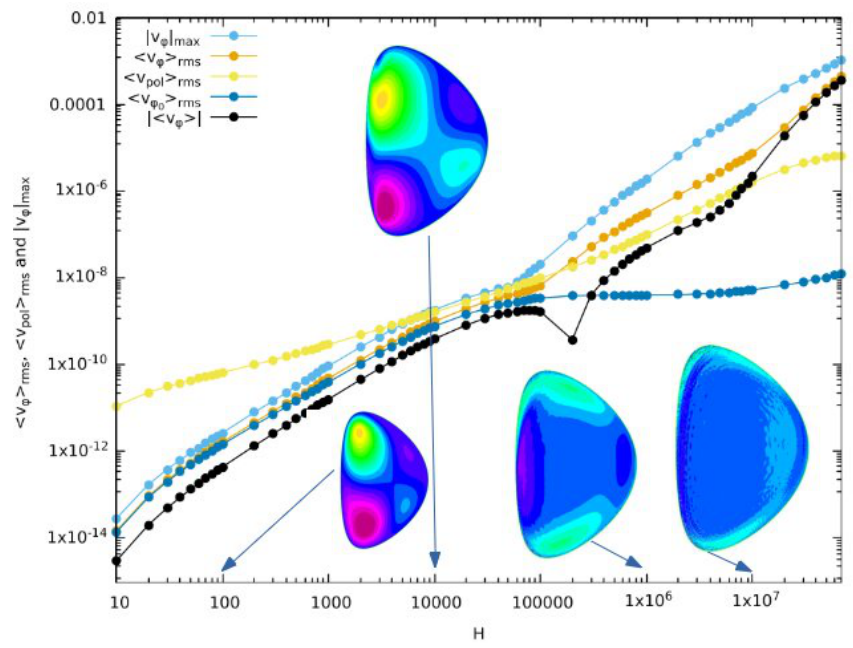

FIG. 9. Same as Figure 8 for the case $\beta(x, y)=0.001 \sin (x-$ 1) $\sin (2 y)$.

We notice that the form of $\beta(x, y)$ influence much the topology of the toroidal velocity field at low Hartmann numbers where the effect of viscosity is dominant. For instance, when $\beta(x, y)$ does not depend on $y$, like in the $f_{1}$ or $\beta(x, y)=0$ cases, the symmetry properties with respect to $y$ presented in Sec. IIC are preserved, and 
there exists an odd solution for the toroidal velocity field. Yet, as it is clear from the comparison between the insets of Figure 8 and Figs. 3 and 4, the modification in the boundary conditions induces a change from two pairs of contra-rotating vortices when there is no magnetic perturbation to a single up-down pair of contra-rotating vortices for the form $f_{1}$ of the magnetic perturbation. The root-mean-square value of $v_{\varphi}$ is larger in the later case than for $\beta(x, y)=0$ and the discrepancy increases with the Hartmann number as seen on Fig. 8.

In the case of external magnetic perturbations with the $f_{3}$ form, the oddness of $f_{3}$ with respect to $y$ contradicts the natural even character of the $u_{5}$ variable and breaks the symmetry properties of the system. A consequence is depicted on Fig. 9: there is now a clear non-vanishing net toroidal flow. This effect is more pronounced for the case $\beta=0.001 \sin (2 y)$ than for $\beta=0.001 \sin (x-1) \sin (2 y)$ since the effective amplitude of the symmetry breaking in $y$ is larger in the former case (because we have here $|\sin (x-1)|<1)$. Features of the toroidal velocity field are presented in Figures 10, 11 and 12. They clearly

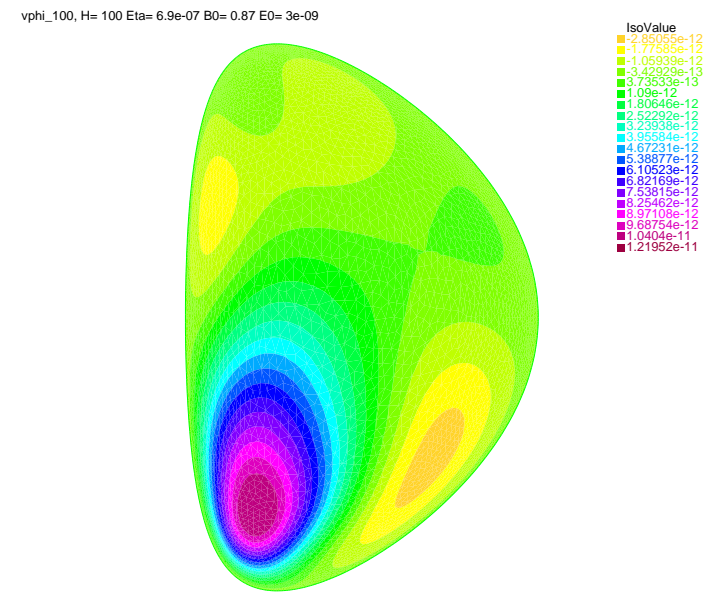

FIG. 10. Toroidal velocity field computed computed with the Finite Element Method (FEM) using FreeFem ++ for $H=$ 100 for $E_{0}=3 \times 10^{-9}, \eta=6.9 \times 10^{-7}$, and $B_{0}=2.8 \mathrm{~T}$ in JET geometry with $\beta(x, y)=0.001 \sin (2 y)$ in (8).

show that $v_{\varphi}$ is no longer odd in $y$ so that there is a net mass flow of the tokamak plasma. This is confirmed by the behaviour with respect of the Hartmann number of the absolute value of the average toroidal velocity $\left|\left\langle v_{\varphi}\right\rangle\right|$ that is markedly above the ones plotted in the $y$ symmetric $\beta(x, y)=0$ and $\beta(x, y)=0.001 \sin (x-1)$ cases in Figs. 6 and 8 and is of the order, or so, of the root-mean-square toroidal velocity.

Finally, the influence of the amplitude of the magnetic perturbation was tested by varying the amplitude $h$ for a given form of the perturbation. We consider here the form $f_{2}=\sin (2 y)$. Figure 14 presents the results. As $h \rightarrow 0$, one recovers the behaviour of $\left\langle v_{\varphi}\right\rangle_{\mathrm{rms}}$ in the absence of magnetic perturbation. As $h$ increases, the curve $\left\langle v_{\varphi}\right\rangle_{\mathrm{rms}}(H)$ takes off the $h \rightarrow 0$ curve above some $H$ value

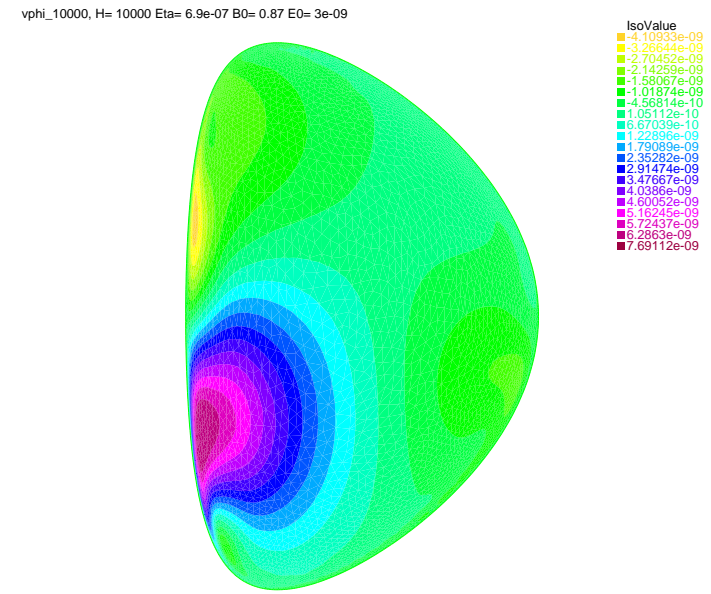

FIG. 11. Same as Figure 10 for $H=10^{4}$.

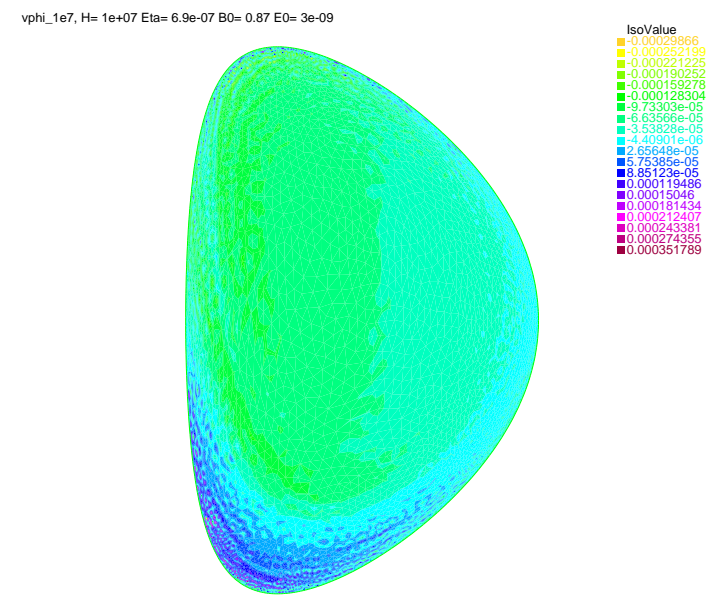

FIG. 12. Same as Figure 10 for $H=10^{7}$.

that decreases when the amplitude $h$ increases.

\section{RESULTS FOR REALISTIC PARAMETERS AND DISCUSSION}

The above results clearly indicate that the introduction of some external vacuum magnetic perturbation contradicting the even character of the magnetic flux potential function $\left(u_{5}\right.$ in the present system), e.g. by having the form of an odd function in $y$, allows to break the natural up-down symmetry properties of the steady-state viscoresistive MHD system (4). In particular, this breaks the odd character with respect to the horizontal midplane of the toroidal velocity field which induces a non-vanishing net toroidal flow of the tokamak plasma. Let us stress here that the non-zero average of the toroidal velocity obtained in the cases without up-down symmetry breaking is a numerical artifact that can be diminished by increasing the resolution or by considering P2 instead of $\mathrm{P} 1$ elements in the finite element computations. Using 


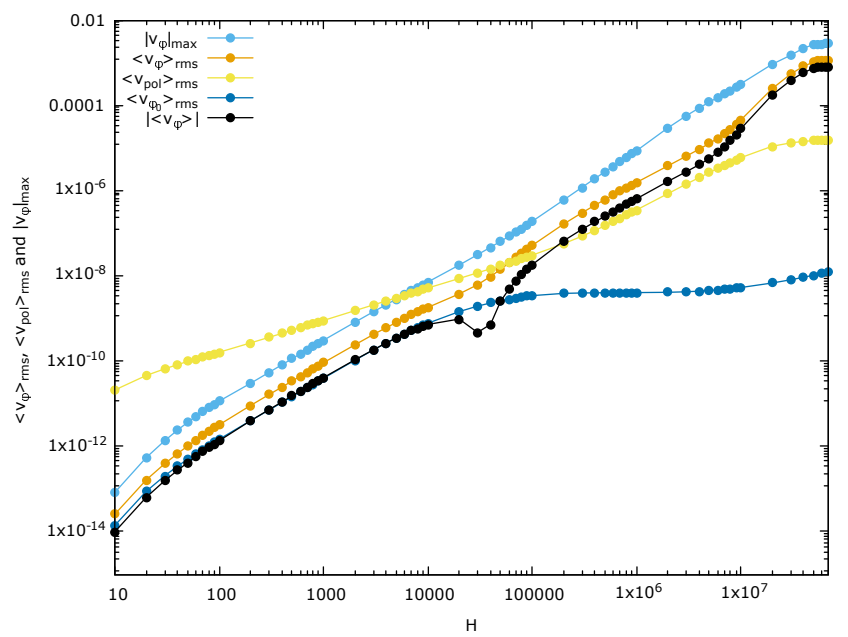

FIG. 13. Maximal absolute value of the toroidal component of the velocity, $\left|v_{\varphi}\right|_{\max }$, and some section-averaged quantities: root-mean square of the toroidal and poloidal velocity, $\left\langle v_{\varphi}\right\rangle_{\mathrm{rms}}$ and $\left\langle v_{\mathrm{pol}}\right\rangle_{\mathrm{rms}}$, absolute value of the average toroidal velocity $\left\langle v_{\varphi}\right\rangle$ associated to the steady flow as a function of the Hartmann number in Alfvèn velocity $v_{\mathrm{A}}$ units for $\beta(x, y)=0.001 \sin (2 y)$ in $(8)$.

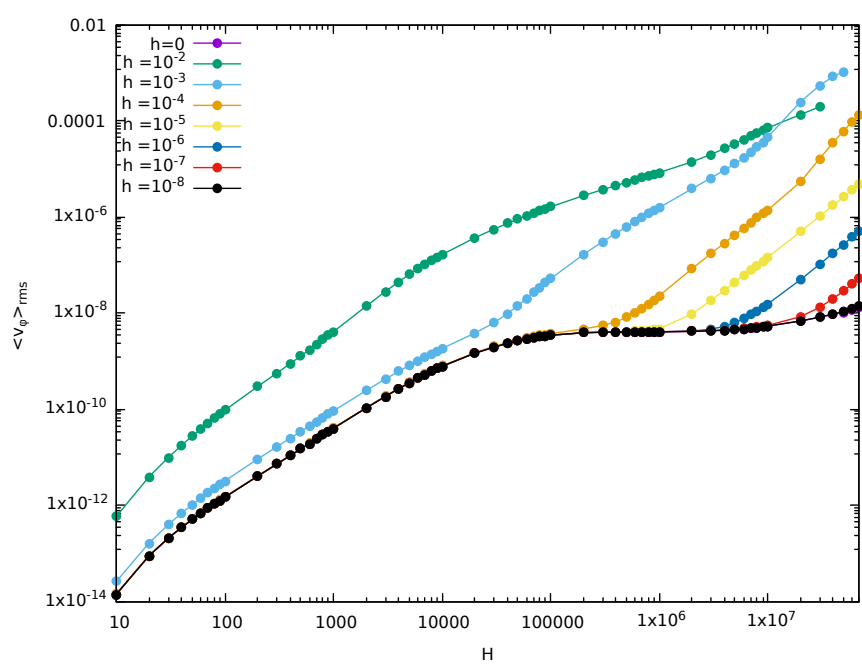

FIG. 14. Root-mean square of the toroidal velocity $\left\langle v_{\varphi}\right\rangle_{\mathrm{rms}}$ as a function of the Hartmann number in Alfvèn velocity $v_{\mathrm{A}}$ units for $\beta(x, y)=h \sin (2 y)$ and various amplitudes $h$.

P2 elements, we observed that the average of the toroidal velocity, $\left\langle v_{\varphi}\right\rangle$, is numerically decreased by two orders of magnitude below that computed with $\mathrm{P} 1$ elements without modifying the rms values. However, this procedure has a high numerical cost and becomes too prohibitive at very large Hartmann numbers.

The numerical results presented in Sec. III have been obtained for the value of the dimensionless resistivity $\eta=6.9 \times 10^{-7}$. Even if this is a large value (larger than the one expected in $\mathrm{JET}^{4}$ ), they give a proof of principle that, all other things being equal, substantially larger magnitudes of the poloidal and toroidal steadystate speeds can be reached with respect to the case without magnetic perturbation (corresponding to a limiter configuration with a perfectly conducting wall). Let us note here that the $f_{2}$ form of magnetic perturbation clearly breaks the up-down symmetry but is not supposed to be an optimal solution for this breaking.

It is important to investigate the evolution of those results under more realistic values of resistivity. Therefore, we ran numerical simulations by decreasing $\eta$ by two orders of magnitude down to $\eta=6.9 \times 10^{-9}$ and present now the results for the corresponding tokamakrelevant set of physical parameters. Simulations were done with P1b finite elements to improve numerical robustness. We used a small magnetic perturbation with $\beta(x, y)=0.04 \sin (2 y)$ yielding the perturbed magnetic flux function represented on Figure 15. Because the value of the equilibrium poloidal magnetic field is now realistic, being much larger than for the results obtained in Section III, the value of the amplitude $h$ of the perturbation in $\beta(x, y)$ needs to be larger to obtain perturbations of the magnetic flux function similar to that obtained in Section III. As depicted on Figure 16, the effect of the up-down

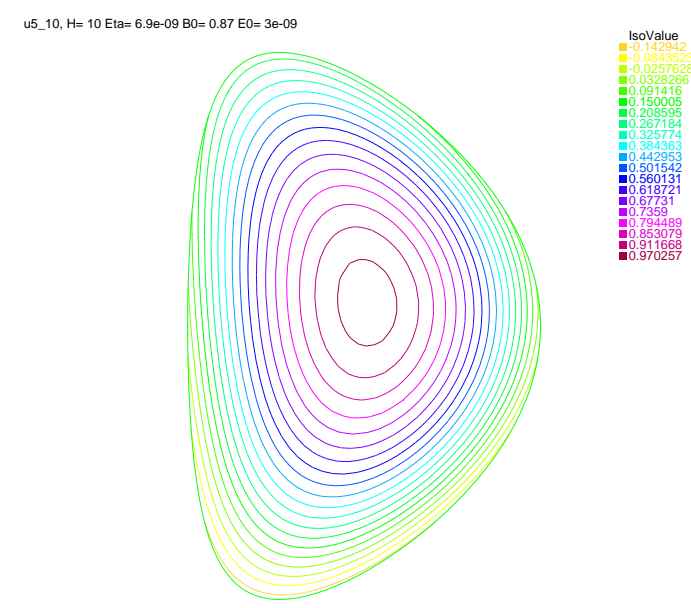

FIG. 15. Contour plot of the magnetic flux function $\chi$ at $H=10$ for $E_{0}=3 \times 10^{-9}, \eta=6.9 \times 10^{-9}$, and $B_{0}=2.8 \mathrm{~T}$, in the case $\beta(x, y)=0.04 \sin (2 y)$. $\chi$ is normalized to one on the magnetic axis.

symmetry breaking external magnetic perturbation is to trigger a net toroidal flow and enhance the rms value of the toroidal velocity with respect to the non-perturbed case. One observes the same numerically-driven enhancement of the net toroidal flow as $H$ increases in the latter case. As previously, larger speeds could be attainable with larger magnetic perturbations, yet numerical stability declines as the Hartmann number increases so that we keep to the perturbation depicted on Figure 15 .

Let us finally examine the impact of the no-slip assumption for the toroidal velocity field in boundary conditions. One may indeed argue that, in the case when the plasma is not in contact with the tokamak walls, but 


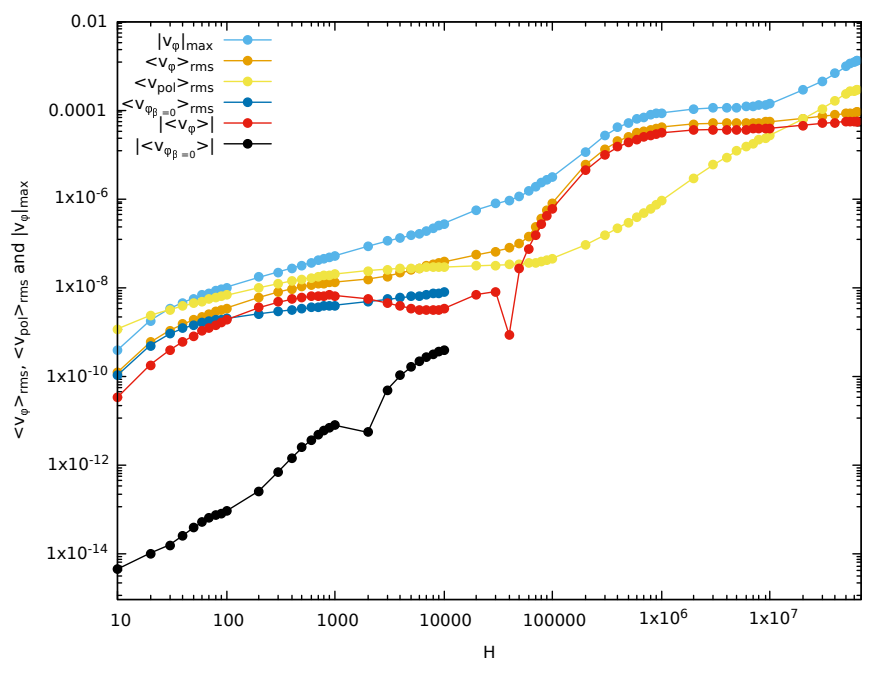

FIG. 16. Root-mean square of the toroidal and poloidal velocities and maximal value of the modulus of the toroidal velocity as a function of the Hartmann number in Alfvèn velocity $v_{\mathrm{A}}$ units for $E_{0}=3 \times 10^{-9}, \eta=6.9 \times 10^{-9}$, and $B_{0}=2.8 \mathrm{~T}$ in the cases $\beta(x, y)=0.04 \sin (2 y)$ with the boundary conditions (8). For reference, the root-mean-square and average of the toroidal velocity is also represented for $\beta(x, y)=0$.

is surrounded by vacuum, the distortion of flux surfaces induced by some external magnetic perturbations (see Figures 7 and 15) would create some small leakage of plasma so that the plasma domain $\Omega$ would realistically be surrounded by a halo plasma instead of vacuum. This questions the assumption of full-confinement state that is implicit in the vanishing of the normal and toroidal components of the velocity field on $\partial \Omega$. Consequently, we tested the impact of replacing the condition $u_{4}=0$ reflecting the vanishing of the toroidal velocity field on $\partial \Omega$ by a more general Robin-type boundary condition of the form $a u_{4}+b \partial_{n} u_{4}=g$ where $a, b$ and $g$ are functions defined on $\partial \Omega$ and $\partial_{n}$ denotes the normal derivative with respect to $\partial \Omega$. One such boundary condition with a physical meaning is $\partial_{n}\left(u_{4} / r^{2}\right)=0$. This amounts to a free-slip boundary condition when the toroidal angular velocity has normal to wall derivative equal to zero. The results are numerically stable. For the computed values of the Hartmann number, they indicate that the influence of the choice of the boundary condition on the toroidal velocity $\left(u_{4}\right)$ on the poloidal steady-state velocity is weak. This is also the case for low and moderate values of the Hartmann number on the toroidal steady-state plasma speed. However, there exists a clear enhancement of the magnitude of the toroidal velocity at large, physically relevant, Hartmann values in the case of the free-slip toroidal boundary condition $\left(\partial_{n}\left(u_{4} / r^{2}\right)=0\right)$ compared with the no-slip toroidal boundary condition $\left(u_{4}=0\right)$. Considering the toroidal no-slip results of Figure 16, maximal values of the toroidal speed reach some units of $\mathrm{km} . \mathrm{s}^{-1}$ for $H \simeq 5 \times 10^{7}$ for the up-down symmetry-breaking $\beta(x, y)=0.04 \sin (2 y)$ at $\eta=6.9 \times 10^{-9}$ corresponding to the disturbed magnetic flux function depicted on Fig-

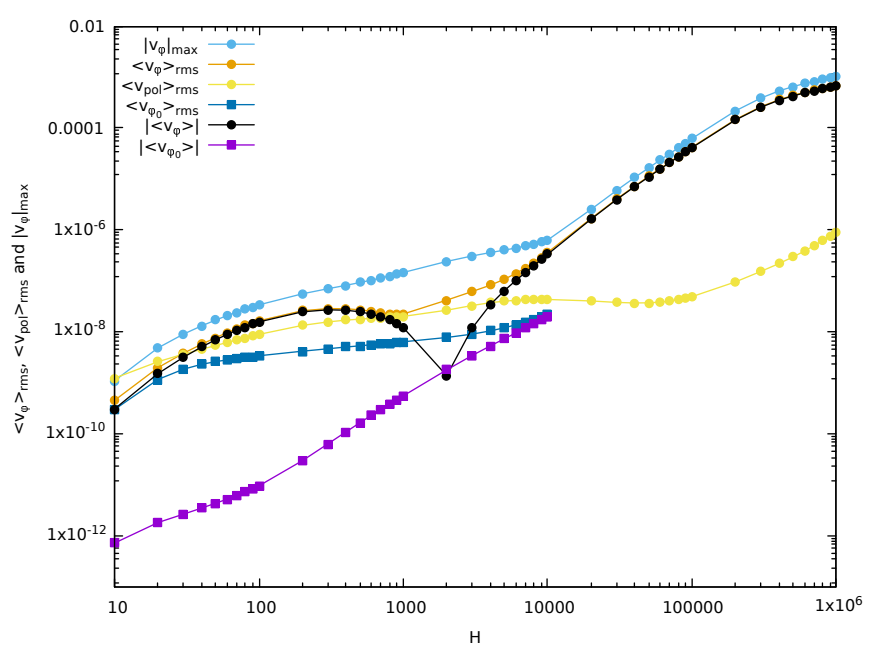

FIG. 17. Same as Figure 16 with the boundary condition $\partial_{n}\left(u_{4} / r^{2}\right)=0$ instead of $u_{4}=0$ in (8).

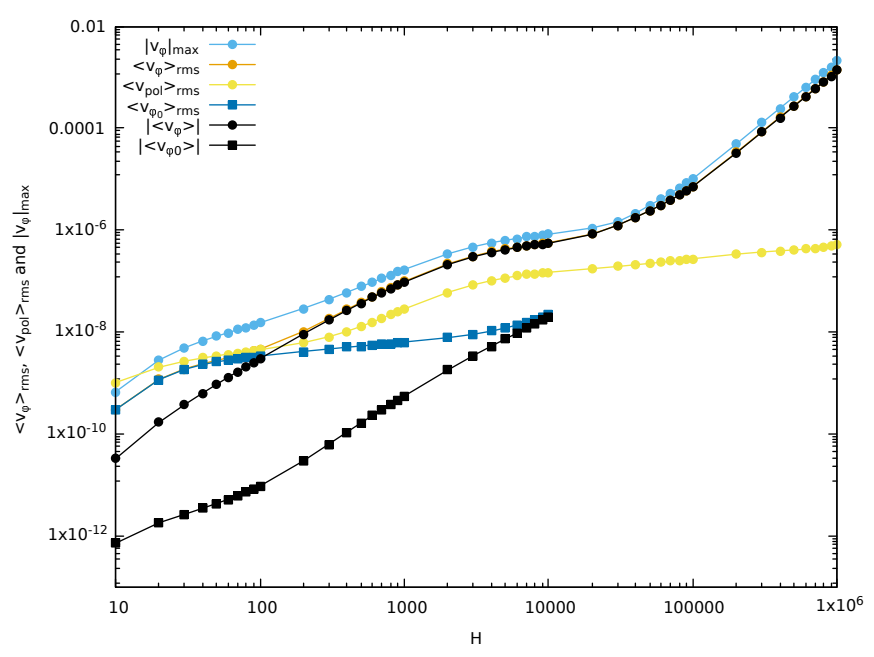

FIG. 18. Same as Figure 17 with a ten times smaller function $\beta(x, y)$, namely $\beta(x, y)=0.004 \sin (2 y)$.

ure 15. Yet, Figure 17 shows that, at $H=10^{6}$, the rms toroidal velocity is one order of magnitude higher than the one obtained for the same Hartmann number in the toroidal free-slip case. Even more, Figure 18 gives at large $H$ the same order of toroidal speeds than in Figure 17 although the external magnetic perturbation is one order of magnitude smaller. This is a noticeable result with interesting perspectives in terms of fusion applications. Figure 19 depicts the toroidal velocity field at $H=100$ for both no-slip and free-slip toroidal boundary conditions.

\section{CONCLUSION AND PERSPECTIVES}

The present academic study shows that axisymmetric (i.e. $n=0$ ) external magnetic perturbations can break the up-down natural symmetry of the visco-resistive 

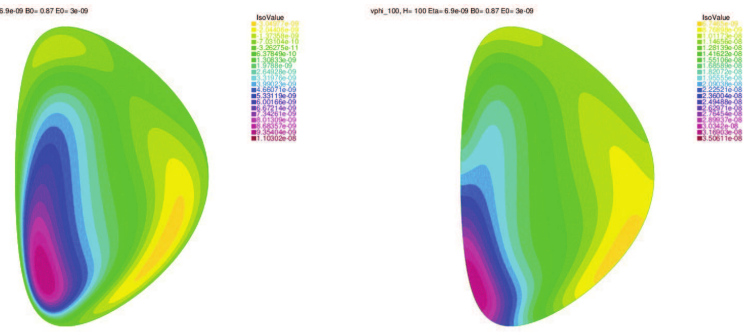

FIG. 19. Toroidal velocity field at $H=100$ for $E_{0}=3 \times$ $10^{-9}, \eta=6.9 \times 10^{-9}$, and $B_{0}=2.8 \mathrm{~T}$ in the cases $\beta(x, y)=$ $0.04 \sin (2 y)$ with (left) $u_{4}=0$; (right) $\partial_{n}\left(u_{4} / r^{2}\right)=0$ in Eqs. (8).

MHD equations and produce a net axisymmetric tokamak plasma steady flow with potentially relevant applications to magnetic confinement fusion. In the case of toroidal free-slip boundary condition, that may be relevant in the realistic case when the plasma is surrounded by a halo plasma, interestingly large steady-state toroidal speeds are obtained at high Hartmann numbers.

Although some physically realistic parameters were used, there are various, more or less numerically demanding, perspectives to this work in order to get a closer application to experiments. Some of them are listed below.

(i) A fully realistic description would obviously mean to consider three-dimensional steady states which would request a considerably higher numerical cost. Yet, by construction, tokamaks are close to axisymmetry making meaningful to consider the KAM-like approach with a zero-order axisymmetric state and higher-order threedimensional perturbations. Moreover, it is meaningful to restrict on axisymmetric $2 \mathrm{D}$ steady-states to find ways to promote large plasma speeds without feeding deleterious $3 \mathrm{D}$ effects such as magnetic braiding ${ }^{13}$.

(ii) Plasma density is not constant in tokamaks but decreases close to its border. This inhomogeneity calls for a reconsideration of the incompressibility assumption. Allowing the plasma to be compressible would substantially complicate the numerical resolution. Besides, the space dependence of plasma density means that one needs to rescale velocities in terms of a local Alfvèn speed. In the present study, plasma density is constant and speeds are given in Alfvèn speed units, meaning that they are Alvèn Mach numbers. It may be that, in the present study, plasma velocities (in $\mathrm{km} . \mathrm{s}^{-1}$ units) are underestimated in the tokamak border region and that their values are indeed larger considering that the local Alfvèn speed is larger there. This point needs to be explored further.

(iii) There remains extra freedom on boundary conditions e.g. on the toroidal vorticity field on which it may be possible to play to enhance plasma speeds. This calls for further investigation.

(iv) Although large plasma speeds are presumably beneficial for plasma confinement, a study on linear stability would be needed to fully characterize the steady-states presented here.
Finally, the generalization of the 2D axisymmetric equilibrium Grad-Shafranov equation to non-vanishing plasma flow has been addressed in this study. The magnitude of the plasma speeds has been shown to increase with the Hartmann number when the up-down tokamak symmetry is broken. Ways to improve the robustness of the numerics at very large Hartmann numbers are under current investigation. The present results yet indicate that some $n=0$ perturbations of the magnetic configuration may help producing $n=0$ steady-state speeds possibly large enough to positively impact confinement for the high, fusion-relevant, values of the Hartmann number.

\section{ACKNOWLEDGMENTS}

Fruitful discussions with N. Minesi, T. Bonnet, R. Guillot, E. Roverc'h and A. Salhi and useful comments by anonymous Referees are gratefully acknowledged. HO thanks the University of Tunis El Manar and the Ministry of Higher Education and Scientific Research of Tunisia for funding. This work has been performed in the frame of the FR-FCM (Fédération nationale de Recherche Fusion par Confinement Magnétique - ITER).

\section{DATA AVAILABILITY STATEMENT}

The data that supports the findings of this study are available within the article.

${ }^{1}$ J. Rice, A. Ince-Cushman, J. Degrassie, L.-G. Eriksson, Y. Sakamoto, A. Scarabosio, A. Bortolon, K. Burrell, B. Duval, C. Fenzi-Bonizec, et al., "Inter-machine comparison of intrinsic toroidal rotation in tokamaks," Nuclear Fusion 47, 1618 (2007)

${ }^{2}$ S. Friedlander and M. M. Vishik, "On stability and instability criteria for magnetohydrodynamics," Chaos: An Interdisciplinary Journal of Nonlinear Science 5, 416-423 (1995), https://doi.org/10.1063/1.166112

${ }^{3} \mathrm{D}$. Montgomery, J. W. Bates, and S. Li, "Toroidal vortices in resistive magnetohydrodynamic equilibria," Physics of Fluids $\mathbf{9}$, 1188-1193 (1997), https://doi.org/10.1063/1.869206

${ }^{4}$ L. P. Kamp and D. C. Montgomery, "Toroidal flows in resistive magnetohydrodynamic steady states," Physics of Plasmas 10, 157-167 (2003), https://doi.org/10.1063/1.1524629

${ }^{5}$ L. P. Kamp and D. C. Montgomery, "Toroidal steady states in visco-resistive magnetohydrodynamics," Journal of plasma physics 70, 113-142 (2004)

${ }^{6}$ J. A. Morales, W. J. T. Bos, K. Schneider, and D. C. Montgomery, "Magnetohydrodynamically generated velocities in confined plasma," Physics of Plasmas 22, 042515 (2015), https://doi.org/10.1063/1.4918774

${ }^{7}$ F. Hecht, "New development in freefem++," J. Numer. Math. 20, 251-265 (2012)

${ }^{8}$ H. Oueslati, T. Bonnet, N. Minesi, M.-C. Firpo, and A. Salhi, "Numerical derivation of steady flows in visco-resistive magnetohydrodynamics for JET and ITER-like geometries with no symmetry breaking," AIP Conference Proceedings 2179, 020009 (2019), https://aip.scitation.org/doi/pdf/10.1063/1.5135482

${ }^{9}$ E. Deriaz, B. Després, G. Faccanoni, K. Gostaf, L.-M. ImbertGérard, G. Sadaka, and R. Sart, "Magnetic Equations with FreeFem++: the Grad-Shafranov Equation \& the Current Hole," ESAIM: Proceedings 32, 76-94 (2011), CEMRACS'10 research achievements: numerical modeling of fusion. Papers 
from the 15th Annual Summer Research Session (Centre d'étude Mathématique de Recherche Avancée en Calcul Scientifique) held in Marseille, July 19-August 27, 2010

${ }^{10}$ The streamfunction $\psi$ is defined up to an arbitrary constant, so that the condition $\psi=$ const on $\partial \Omega$ would be identically valid.

${ }^{11}$ H.-T. Kim, A. C. C. Sips, P. C. de Vries, and J.-E. Contributors,

"Plasma burn-through simulations using the DYON code and predictions for ITER," Plasma Physics and Controlled Fusion
55, $124032(2013)$

${ }^{12}$ L. Spitzer, Physics of fully ionized gases (Interscience Publishers, 1956)

${ }^{13}$ M.-C. Firpo, "Microtearing turbulence: Magnetic braiding and disruption limit," Physics of Plasmas 22, 122511 (2015), https://doi.org/10.1063/1.4938273 